\title{
Comparison of Clinical Effects of Abdominal Aortic Aneurysm Treatment with Open and Endovascular Techniques
}

\author{
Ning Dou ${ }^{1}$, Jing-jing Tan ${ }^{2}$, Jian Zuo ${ }^{3 *}$ \\ ${ }^{1} 1^{\text {st }}$ Department of Surgery, Shanghai Fourth People's Hospital Affiliated to Tongji University, Shanghai 200081, China; \\ ${ }^{2}$ Department of Anesthesia and Perioperative Medicine, Shanghai Fourth People's Hospital Affiliated to Tongji University, \\ Shanghai 200081, China; \\ ${ }^{3}$ Department of Cardiovascular Surgery, First Affiliated Hospital of Air Force Military Medical University of Chinese People's \\ Liberation Army, Xi'an 710032, China
}

Funding: Shanghai Fourth People's Hospital Medicine Discipline Boosting Plan (SY-XKZT-2020-2005); Shanghai Fourth People's Hospital Medicine Discipline Boosting Plan (SY-XKZT-2020-1005).

\begin{abstract}
Objective: To compare the therapeutic effects of endovascular and open surgery on abdominal aortic aneurysms. Methods: From June 2019 to May 2020, 60 cases of abdominal aortic aneurysms (AAA) were divided into observation group (30 cases in endovascular technique group) and control group (30 cases in open technique group). Results: The blood loss, operative time and blood transfusion of the observation group were significantly lower than those of the control group $(P<0.05)$. The incidence of postoperative complications is low, and the incidence of longterm complications is relatively high. Conclusion: In the treatment of abdominal aortic aneurysm, endovascular technology has the advantages of low risk, less trauma, and quick recovery after surgery. Open surgery is suitable for patients who cannot receive endovascular treatment. In order to achieve good treatment effects, it is necessary to choose an appropriate treatment method according to the actual situation of the patient.
\end{abstract}

Keywords: Laparotomy technique; Endovascular technique; Abdominal aortic aneurysm; Clinical

Publication date: November, 2020

Publication online: 30 November, 2020

"Corresponding author: Jian Zuo, zuojian@fmmu. edu.cn

\section{Introduction}

Currently, there are two ways to treat abdominal aortic aneurysms (AAA): surgical repair and endovascular aneurysm repair (EVAR). First, due to the immature endovascular technique, many postoperative complications and uncertain clinical efficacy, EVAR is the only alternative treatment for patients who cannot undergo surgery. AAA refers to the local expansion of abdominal aortic aneurysm and it is a common disease in vascular surgery. If it exceeds the normal value, it can be diagnosed as AAA. With the aging of the population and the deepening of social life, the incidence of the disease is increasing year by year, and people's eating habits are constantly changing. Once an AAA is formed, it is a natural development process, gradually increasing until the tumor ruptures. AAA seriously threatens human life and health. If left untreated, the mortality rate of ruptured abdominal aortic aneurysm is high. The execution of thoracic-abdominal aortic (TAA) replacement surgery was late in China, with fewer cases and lack of relevant research. Currently, there are significant differences between China and foreign countries in epidemiology, etiology and treatment 
history of TAA. The average age of patients is about 40 years old and their life span is long. After connective tissue diseases (such as aortic dissection and Marfan syndrome) occurred, the proportion of TAA patients is higher ${ }^{[1-2]}$. The early treatment effects and long-term prognosis of these patients have always been the concerns of doctors and patients, but these patients have no international treatment experience. Therefore, it is necessary to summarize the experience of thoracic-abdominal aortic surgery in China to provide reference for continuous improvement of surgical efficacy and long-term prognosis. On this basis, 60 cases of abdominal aortic aneurysm were treated with endometrial technique and conventional laparotomy with good results.

\section{Information and Methods}

\subsection{General Information}

From June 2019 to May 2020, 60 patients with subrenal abdominal aortic aneurysms admitted to our hospital were randomly divided into a control group and an observation group, with 30 cases in each group. In the control group, there were 24 males, 6 females, ages ranged from 49-78. In the observation group, there were 22 men and 8 women, ages ranged from 46-76. There was no significant difference in gender and age between the two groups $(P<0.05)$. The study was approved by the hospital ethics committee.

\subsection{Methods}

\subsubsection{Surgery Methods}

The endovascular treatment group enrolled 30 cases of endovascular treatment. The patients underwent general anesthesia and a longitudinal incision of the femoral artery branch. The enlarged image allows the access to knowledge of the size of the tumor, the affected area, and the top and bottom. If the renal artery is not blocked and the tumor disappears, the delivery device can be removed. Thirty patients in the open treatment group received open technique treatment. General anesthesia and laparotomy completely exposed the abdominal aorta. The aorta is effectively blocked under the renal artery. At the distal end of the lesion, the bilateral and abdominal aortas were blocked, the tumor was incised, and the lumbar artery was ligated. Anastomose the branched artificial blood vessel to the proximal end of the abdominal aorta and the two arteries to ensure that blood flow is restored $\mathrm{d}^{[3-4]}$.

\subsubsection{Pre-operative Assessment}

People with high-risk complex AAAs usually suffer from various underlying diseases and require routine preoperative testing. Similar to the preoperative examination, it mainly includes a comprehensive and detailed physical examination, routine biochemical examination and functional assessment of vital organs. Understand the patient's general conditions and physiological functions, and determine whether the patient can tolerate EVAR and the related risks that must be prevented. A comprehensive and detailed physical examination is an important indicator of the severity of the disease. If the patient suffers from severe and persistent abdominal pain or back pain, carefully check for a ruptured aneurysm. Symptoms of hemorrhagic shock include increased heart rate, lowered blood pressure and decreased urine output, requiring careful observation and attention. For example, a sharp drop in hemoglobin is mainly caused by a ruptured aneurysm, while an increase in serum creatinine indicates that the patient has renal failure. It is also necessary to fully understand and evaluate the patient's breathing, circulation, metabolism and other organ functions. If organ function is impaired, the risk of surgery increases. If not handled properly, the function of vital organs will deteriorate further due to surgery, which may eventually lead to severe organ failure or patient failure.

\subsubsection{Post-operative Treatment and Follow-up}

After treatment and follow-up, the patient should lie in bed within 24 hours after the operation, during which vital signs should be continuously monitored. If there are no serious complications after 24 hours, the patient can gradually resume daily activities and reduce the level of treatment. Antibiotics and anticoagulants should be given within 72 hours after surgery to avoid graft infection and thrombosis. Longterm oral aspirin (such as atherosclerosis, coronary heart disease and other diseases) can be considered in combination with clopidogrel, and patients with hypertension, diabetes and other diseases should also be given appropriate drug treatment.

Follow up every 1-3 months. Use ultrasound, CTA or MRA to check for complications. The prognosis and clinical efficacy of patients were evaluated based on the follow-up results. If complications were found, the reasons should be analyzed and corresponding 
measures should be taken as soon as possible. If there were no related complications, the follow-up time can be gradually extended to 6 to 12 months.

\subsection{Statistical Analysis}

SPSS 18.0 software was used to analyze the research data. The measurement data is expressed in $(\bar{x} \pm s)$, and the $\mathrm{R}$ test is used for comparison; the kappa test is used for the comparison of count data, and the difference is statistically significant $(P<0.05)$.

\section{Results}

\subsection{Surgical Results}

Analysis of the surgical status of the two groups of patients: When analyzing the surgical status of the patients, the operative time, blood loss volume, intraoperative blood loss and intravascular admission time were significantly lower than the open group, and the difference between the two groups was statistically significant $(P<0.05)$, as shown in Table 1. Analysis of surgical success rate: In the analysis of surgical success rate, all operations were successful without aneurysm related rupture and no deaths.

Table 1. Comparison of Surgical Status

\begin{tabular}{|c|c|c|c|c|}
\hline Group & $\begin{array}{l}\text { Operative Time } \\
\text { (h) }\end{array}$ & $\begin{array}{c}\text { Admission Time } \\
\text { (d) }\end{array}$ & $\begin{array}{c}\text { Intraoperative Blood Loss } \\
(\mathrm{mL})\end{array}$ & $\begin{array}{l}\text { Blood Loss } \\
(\mathrm{mL})\end{array}$ \\
\hline Endovascular & $172.8 \pm 70.3$ & $15.6 \pm 2.6$ & 0 & $109.3 \pm 27.9$ \\
\hline Open & $290.7 \pm 52.6$ & $30.8 \pm 4.7$ & $1119.2 \pm 259.8$ & $1149.3 \pm 98.5$ \\
\hline$T$ & 15.952 & 12.513 & 18.260 & 20.074 \\
\hline$P$ & 0.001 & 0.001 & 0.001 & 0.001 \\
\hline
\end{tabular}

\subsection{Postoperative Treatment}

The postoperative treatment of the two groups of patients is basically the same, and appropriate antiinflammatory and anticoagulant treatments were required. Imaging examinations and other related examinations should be performed before discharge. The examination results determine whether the patients meet the conditions for discharge. Some patients in the observation group have systemic or femoral artery stenosis, so appropriate anticoagulation therapy should be given to prevent thrombosis. If serious complications occur before and after the operation, they must be treated in time to avoid affecting the patient's clinical efficacy. In the observation group of this study, severe lumen leakage was found after re-examinations. The dynamic monitoring method was chosen because there was no obvious lumen leakage in both groups. In the other group, femoral artery pseudoaneurysms developed and disappeared after local compression therapy.

\section{Discussions}

In recent years, due to the continuous improvement of interventional equipment and endovascular technology, the incidence of postoperative complications has been greatly reduced, and a series of clinical randomized controlled studies have also confirmed that the clinical efficacy of EVAR is not lower than that of normal AAA patients. In addition, EVAR has the advantages of low trauma, quick recovery and low risk, and is gradually replacing surgery as the first choice for AAA patients. On the other hand, there are also a large number of AAA patients with high risk factors or complex anatomy. Due to its inherent high-risk factors or complex anatomy, patients with these complex high-risk factors are twice as difficult and risky to treat as ordinary AAA patients. Patients with high-risk and complex AAA should choose EVAR treatment, and their clinical efficacy should be evaluated through scientific evaluation of treatment risks. This is also a premise for clinical promotion of EVAR in the treatment of high-risk and complex AAA patients in the future ${ }^{[5-6]}$.

Patients with AAA usually have no obvious autonomic symptoms and discomfort in the early stages. As the disease progresses, aneurysms gradually increase, and some people experience symptoms such as bloating, abdominal pain, or back pain. Few patients have symptoms of visceral or lower extremity arterial embolism due to thrombosis in the aneurysm wall. Some people have gastrointestinal and biliary tracts due to compression of aneurysms in surrounding organs and obstruction of the ureters. Due to occult and non-characteristic symptoms, only a few patients encountered a 
pulsating abdominal mass during self-examination or physical examination and went to the hospital for treatment. Before seeking treatment, more patients will ignore this condition or wait for it to become more serious. As clinicians lack experience, more patients will be misdiagnosed. When an aneurysm ruptures, the consequences are catastrophic ${ }^{[7-8]}$.

Pathologically, the abdominal aortic aneurysm is considered to be larger than the normal aortic diameter. Related clinical studies have shown that the growth rate of abdominal aortic aneurysm is directly proportional to the risk of rupture, and the rupture strength is closely related to the diameter of the aneurysm at the time of diagnosis. When an abdominal aortic aneurysm forms in the patient's body, it will continue to grow until it ruptures, seriously threatening the patient's life. Open technique therapy and endovascular therapy can be applied clinically. The traditional laparotomy is effective, but due to the many complications before and after the operation and the high mortality rate, it is not suitable for patients with many complications and weak physique. The application of endovascular technique in the treatment of abdominal aortic aneurysm is mainly to use abdominal aortic CTA to detect aneurysms. Placing a stent graft with appropriate size in the aorta can effectively separate the blood flow from the tumor wall, ensure that the blood in the stent graft is unblocked, and effectively maintain the unblocked blood flow in the abdominal aorta. There are few treatment complications and little trauma, which can guarantee the patient's treatment effects.

The advantages of abdominal aortic aneurysm surgery are low trauma and short postoperative recovery time. It is mainly suitable for elderly patients. Open surgery is suitable for patients who cannot receive endovascular treatment, while ordinary patients are young and have fewer complications. In order to achieve good treatment effects, it is necessary to choose an appropriate treatment method according to the actual situation of the patient.

\section{References}

[1] Li M, Zhou JH, Zhong QL, et al. Analysis of medium to longterm therapeutic effect of endovascular repair of abdominal aortic aneurysm [J]. Shanxi Medical Journal, 2020, 49(17): 2276-2278.

[2] Liu X, Dai YB. Research progress in diagnosis and treatment of type II endoleak after endovascular repair of abdominal aortic aneurysm [J]. Journal of Modern Medicine \& Health, 2020, 36(15): 2412-2415.

[3] Zhou W, Cao WD, Zhou JP, et al. Analysis on the Efficacy and Prognosis of Endovascular Repair in the Treatment of Elderly Patients with Abdominal Aortic Aneurysm [J]. Clinical Medicine \& Engineering, 2020, 27(8): 1011-1012.

[4] Chen JW. Prevention and management of complications after endovascular repair of abdominal aortic aneurysm [D]. Guangzhou Medical University, 2020.

[5] Liu X. Research progress of type II endoleak after endovascular repair of abdominal aortic aneurysm [D]. Chongqing Medical University, 2020.

[6] Li ZQ, Kong W. Microcalcification: pathological features and mediating factors of abdominal aortic aneurysm [A]. Chinese Physiological Society; Chinese Physiological Society. Comprehensive abstract of the 15th National Youth Outstanding Physiology Academic Paper by the Zhang Xijun Foundation of Chinese Physiological Society; Abstract of Papers of the 13th National Young Physiologist Academic Conference of Chinese Physiological Society [C]. Chinese Physiological Society; Chinese Physiological Society: Chinese Physiological Society, 2019:1.

[7] Guo SL. Analysis of the difference in imaging factors of ruptured and complete abdominal aortic aneurysms [D]. Jilin University, 2019.

[8] Liu Y, Wang YY, Zhao XN, et al. Application value of contrast-enhanced ultrasound in the selection of endovascular treatment strategies for abdominal aortic aneurysms [A]. Chinese Association of Ultrasound in Medicine and Engineering. Paper Collection of the 14th National Conference on Cerebral and Cervical Ultrasonography by the Chinese Association of Ultrasound in Medicine and Engineering[C]. Chinese Association of Ultrasound in Medicine and Engineering.: Chinese Association of Ultrasound in Medicine and Engineering., 2018: 2. 